\title{
Measurement Possibility Analysis of the Real Values of the Tissue Structure Parameters with the Use of Optical Coherent Tomography Method
}

\author{
J. KASPRZAK ${ }^{a, *}$, D. KęCIK ${ }^{a}$ AND A. ZAJAC ${ }^{b}$ \\ ${ }^{a}$ Department and Ophthalmology Clinic, I Medicine Faculty, Medical University of Warsaw \\ W. Lindleya 4, 02-005 Warsaw, Poland \\ ${ }^{b}$ Institute of Optoelectronics, Military University of Technology, S. Kaliskiego 2, 00-908 Warszawa, Poland \\ The issues connected with the usefulness of the method of averaging A-scans applied in measurements of \\ real optical parameters with the use of commercial optical coherent tomography systems have been discussed. \\ The basic method description and examples of applications have been given. Examples of the characteristics of \\ metrological devices used in medical practice as well as the possibility of the method applied to the turbidity \\ assessment have been presented.
}

PACS: 06.20.-f, 42.79.-e, 42.79.Ls, 42.30.Wb, 87.19.rm

\section{Introduction}

A source of an optical signal used for tissue structure analysis in optical coherent tomography (OCT) is the radiation scattered by components of the tissue structure. The interferometry technique applied in OCT devices uses radiation sources characterized by the short coherence path. It permits the intravital measurement of the optical signal from the area determined by the beam focus of the diameter ranging from a few $\mu \mathrm{m}$ to a dozen or so $\mu \mathrm{m}$ and depending on the coherence path. Techniques allowing controlling the length of the optical path in the reference arm of an interferometer make the measurement from the tissue regions located at different depths (towards beam axis) possible $[1,2]$. In the aftermath of this, the extinction profiles far into the tissue (so called A-scans) are gained. In the spectral method developed in recent years A-scans are obtained as a result of the analysis of the spectrum of the optical signal dispersed with the use of diffraction grating [3-5]. Putting together A-scan series obtained as a result of diagnostic beam vertical dislocation along programmed contour allows imaging internal structures - similarly as in the ultrasonography presentation B. Commercially available ophthalmologic devices based on OCT technique are designed for imaging the structures of the eyeball rear pole. The users quickly took an interest in advantages resulting from the use of this technique for imaging the structures others than retina. Among other things attempts at an-

* corresponding author; e-mail: kasprzak@amwaw.edu.pl alyzing the structure of the anterior segment of the eye were made. The research into centres of coagulation in a cornea and an eye lens performed with the use of an erbium laser were carried out successfully [6]. Much more problems were caused by issues requiring a quantitative analysis of an optical signal - occurring when trying to use the OCT technique for the evaluation of the turbidities of both the cornea and the eye lens. Even more problems appeared when trying to use this technique for the evaluation of the processes requiring the determination of the concentration and the size of the scattering centers.

\section{Material and method}

Method assumptions allowing the quantitative analyzing of an optical signal have been developed by means of OCT1 system manufactured by Humfrey company. The device allows the imaging of partly transparent structures with the axial resolution about $15 \mu \mathrm{m}$. The minimum scan length on the retina equals $1.3 \mathrm{~mm}$. The measurement results are displayed in a measuring window with the dimension of $100 \times 500$ pixels and after the user acceptation they are recorded in a table with the same dimension. The device software uses algorithms designed for the structure analysis of tomograms of normalization coefficients for a need to measure retina. Other structure evaluation requires the determination of calibration coefficients taking into consideration the parameter difference of the vertical scanning sector (allowing for eye optics) as well as optical characteristic difference of these structures in comparison with the retina. This issue has 
a basic importance in case of structural analyses and will not be discussed in this contribution.

The most crucial issue was to decide whether the chosen metrological characteristics of the device were enough to evaluate the morphologic features of the retina. Another important thing was to determine whether requirements necessary for the quantitative measurement of an optical signal are fulfilled. The device susceptibility to the interferences is also a very important issue. A source of interferences is factors resulting from the method peculiarity or technical solution quality. Subjective factors resulting from the user experience seem to be also important. Such features as the sample arrangement in measuring area or setting parameters influencing the reproducibility of measurement conditions are evaluated by the user. In case of the research with the use of physical models most subjective factors can be eliminated and others can be controlled. In short- and long-time research on the stability, the homogeneous, strongly scattering planes permitting the easy edge identification were applied. Depending on the needs, the samples both with superficial and special distribution of the scattering centers were used.

In case of the measurement requiring sample shifting, the superficial examination was set perpendicular to the scanning sector axis. The parallel setting of the tomogram edge to the measuring window edge is very important. It simplified the algorithms responsible for the identification of the surface examined. In order to limit the geometrical deformation linear scans of minimum length were applied.

For the sake of big fluctuations the attempts at carrying out metrological characteristics and evaluating the stability of the OCT1 device with the use of the software (delivered by the producer) turned out to be ineffective and required working out other proper methods.

\section{Interference analysis}

Searching for the interference sources the spectral analysis of an optical signal used for the tomogram reconstruction obtained for chosen types of samples (the homogeneous samples characterized by spatial scattering center distribution were used) was conducted. The analysis was done by means of fast Fourier transform $(\mathrm{FFT})(A, n)$ of the Signal Processing Toolbox module from MATLAB platform [7].

For individual samples a series of 16 tomograms (in identical conditions) was performed. The analysis was carried out for the central A-scan from the tomogram chosen from the middle of the series. Before starting the FFT procedure, the average signal value evaluated on the basis of the series of the 16 tomogram was subtracted from the A-scan analyzed. The FFT procedures were repeated for the chosen set of data - after previous median filtration. The comparison results without and with filtration allowed determining the optimum parameters of the filtration procedure. The exemplary results of the spectral analysis performed for six different samples were depicted in Fig. 1. The results obtained show that beside

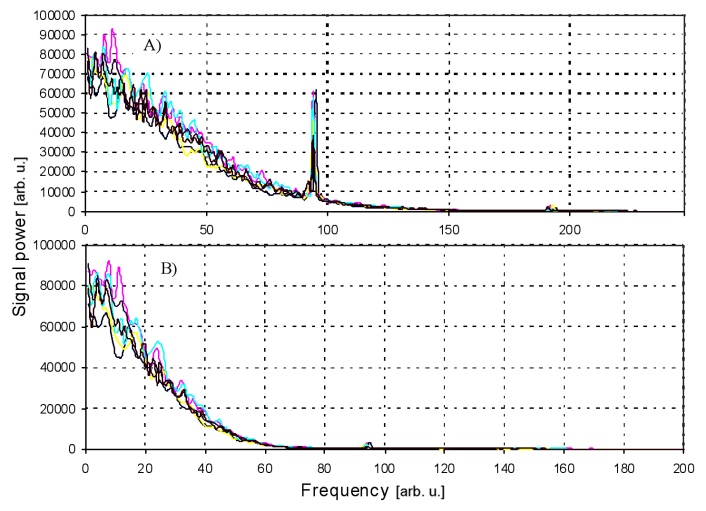

Fig. 1. The optical signal spectrum obtained by means of the FFT method (for 6 samples): the spectrum of tomograms without filtration (A); the spectrum of tomograms with filtration (B). The individual lines (marked with different colours) represent the optical signal spectrum of the samples examined.

the wide optical signal band there is a strong and narrow band of apparatus noises generated in detection process (Fig. 1A).

In case of the OCT1 system, the undesirable band can be easily identified in all tomograms analyzed regardless of the sample type. For STRATUS system such phenomena were not observed.

\section{Fluctuation influence on the evaluation of the structures examined}

In the next stage the fluctuation influence of the optical signal on the identification process of the objects with considering the structure surface in particular was analyzed. The example illustrating this issue for the sample characterized by spatial distribution of scattering centers was depicted below. The scattering profiles for the A-scan obtained from the central part of the scanning line (A-scans with the number of 50 were chosen from 10 tomograms) were recorded at $30 \mathrm{~s}$ intervals. A very good location stability of an optical signal increase in successive recordings was observed. In this area the location identification of the rear surface of the sample examined was possible (Fig. 2). After averaging the tomogram series analyzed the standard deviation for the optical signal represented by chosen A-scans was calculated (Fig. 3). A sudden increase in the fluctuation in the area corresponding to the sample surface is very characteristic.

The fluctuation importance in the edge identification process is presented in the setting-up of normalized characteristics including the optical signal average value for the A-scan group presented, standard and relative deviations. The relative deviation was calculated by dividing the standard deviation values for consecutive coordinate determined in beam axis direction by the average optical 


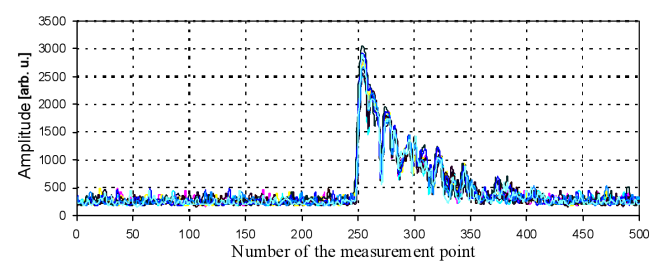

Fig. 2. The setting-up of the A-scans selected from the 10 -tomogram series performed at $30 \mathrm{~s}$ intervals for the samples characterized by spatial distribution of scattering centers.

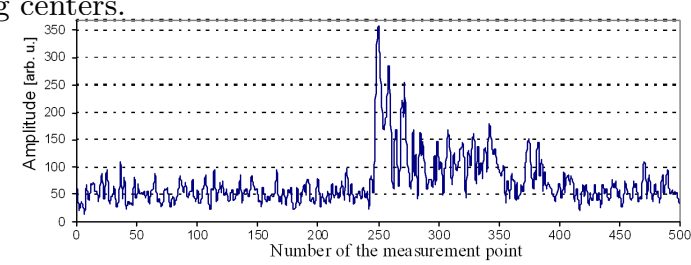

Fig. 3. The standard deviation of the A-scans group depicted in Fig. 2.

signal value in this point. The results of the comparative analysis are presented in Fig. 4.

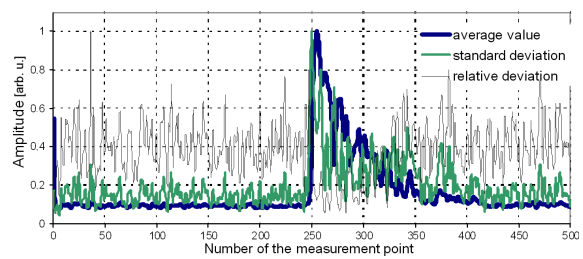

Fig. 4. Normalized curves of the average value of standard and relative deviations for the A-scans series presented in Fig. 2.

The results obtained show that the standard deviation sharply rises in the area corresponding to the sample edge. Despite the optical signal fluctuation increase in this region, the relative value of the standard deviation within the edge decreases. The edge identification procedure of the structures analyzed may be successfully realized on the basis of individual A-scans.

For the sake of the significant increase in the optical signal variability in this region, the use of single A-scans for the optical parameter measurement of the sample leads to obtaining results with low statistical variation. The additional fluctuation source may be the density change of scattering centers resulting from the location instability of the measuring point within inhomogeneous regions of the sample.

\section{Procedures of the measurement of an optical signal amplitude}

Much better repeatability of the optical signal measurement is observed after applying the so-called averaging A-scans method. In this method the optical signal values for individual points of the averaging A-scan are calculated by means of the value averaging (perpendicularly to a scanning sector axis) of an A-scan series of the tomogram analyzed. The averaging procedure may be successfully simplified applying flat test plates, short scans, and the perpendicular setting of the scanning sector to the plate surface. Then the tomogram edge corresponding to the test plate surface is parallel to the measuring window edge. As a result, the conditions allow one applying the averaging procedure for over 100 A-scans. As it was expected, even 10 times drop of fluctuation (independently of the sample type) is achieved. Exemplary results for the samples characterized by spatial scattering centers distribution are shown in Fig. 5 and Fig. 6.

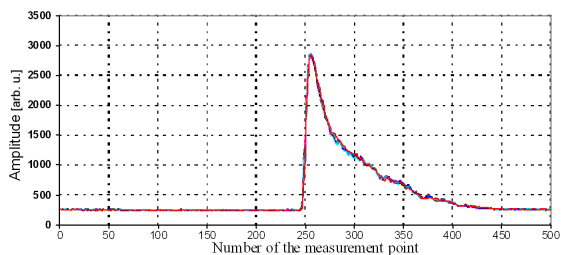

Fig. 5. The averaged A-scans for the series of 10 tomograms for the sample characterized by the spatially located scattering centers. The stability of both the location of the sample edge and the average scattering values is noticeable. In maximum the average value for this series equals $2853 \pm 11$ (in conventional units). The relative uncertainty equals $0.4 \%$.

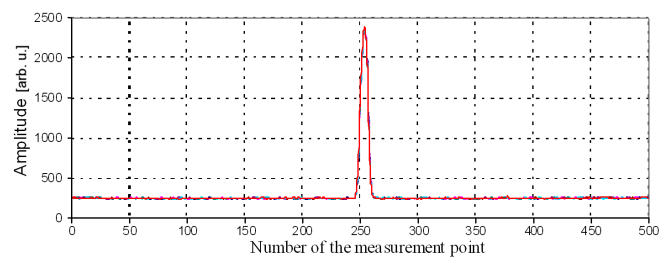

Fig. 6. The setting-up of the averaged A-scans for the series of 10 tomograms for the sample characterized by the superficial distribution of scattering centers. The maximum value of the averaged A-scans equaled $2361 \pm 9$.

For the sake of the considerable fluctuation decrease, the method of averaged A-scans is an effective and simple tool allowing evaluating the metrological characteristics of a given apparatus in clinical conditions.

\section{The analysis of the results}

The method of averaged A-scans was applied among other things to determine the chosen characteristics of devices applied in the clinic (for OCT1 and STRATUS systems). Using the method mentioned above for OCT1 system assessment, a lot of problems making it impossible to measure the optical signal real parameters both in 
tissues and model systems were stated. The heterogeneity of the measuring eye sensitivity as well as the variation of this characteristic revealed at each switching-on have a crucial importance here. The area of the first 100 points of the measuring window is a potential source of artefacts. In case of tomogram location in this area the apparent edges are created. A long period (many hours) required to achieve the relative device stability is the additional difficulty (Fig. 7).

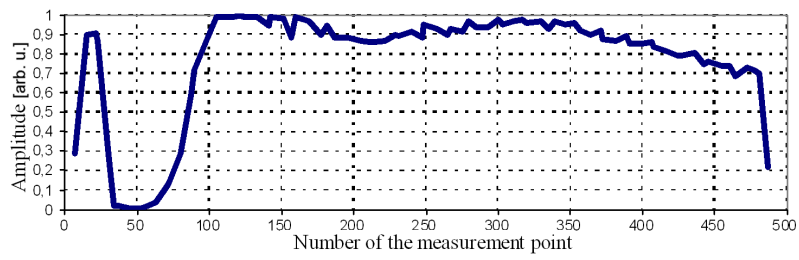

Fig. 7. The exemplary sensitivity characteristic for the full range of the OCT1 device measuring window.

The STRATUS system showed in this respect much better characteristics (Fig. 8). For this device the tomogram consists of $515 \mathrm{~A}$-scans. Individual A-scans include 1024 measurement points distributed uniformly in the axial direction. The minimum scan length on the retina equals $5 \mathrm{~mm}$. The analyses were done using the result of the average value of $100 \mathrm{~A}$-scans from the central tomogram part.

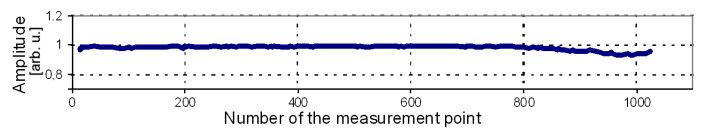

Fig. 8. The exemplary characteristic of the measuring window sensitivity performed for STRATUS device.

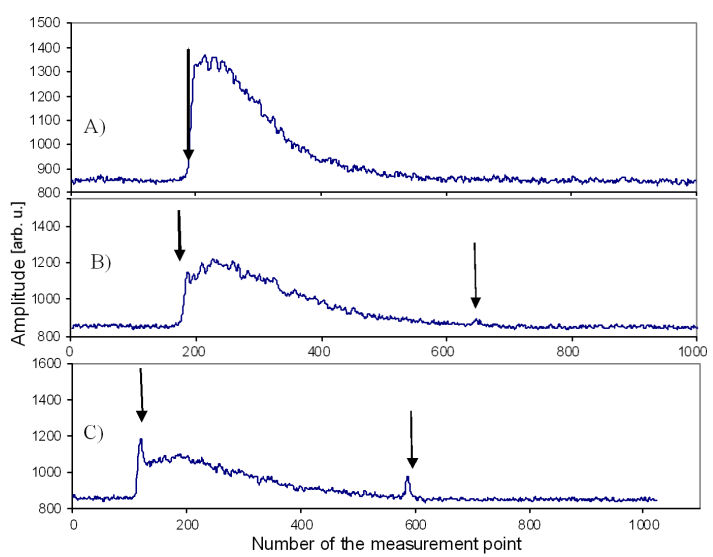

Fig. 9. The optical signal change in the turbid medium depending on the dilution. (A) Non-diluted milk, (B) diluted milk in $1 / 10$ ratio, $(\mathrm{C})$ diluted milk in $1 / 30$ ratio. The internal surfaces of the vessel were marked by means of arrows.
High stability and high homogeneity of the characteristic of the measuring window sensitivity (for STRATUS system) allow us conducting the structural analysis of the tissues examined practically in the whole accessible area of the measuring window. During the research, a several percent's sensitivity decrease of the measuring window within the area of 200 last points did not noticeably influence proceed of the structural analyses of the tissues examined. In case of quantitative examinations it is advisable to avoid this area. The tomogram analyzed should be located in the area including the first 800 points. The example of this technique usage for the optical signal analysis in turbid medium (in the form of milk) depending on the dilution is presented in Fig. 9.

The curves of the optical signal presented in Fig. 9 were obtained analyzing (using the averaged A-scans method) the tomograms performed by the STRATUS device. The milk in glass phial without and with dilution $(1 / 10$ and $1 / 30)$ was examined. The thickness of the layer examined was $949 \mu \mathrm{m}$. For the samples characterized by lower turbidity (Fig. 9A,C) it is easy to point at the region corresponding to the reflection from internal vessel surfaces. The building up of the optical signal in the external layer of the front surface is also very characteristic of this phenomenon. This layer thickness increases for the samples more diluted.

\section{Summary}

The method of the averaged A-scans, as a result of the optical signal fluctuation decrease, facilitates the evaluation of the selected metrological parameters of the OCT devices in clinical conditions. Thanks to an increase in the statistical variation, it is possible to perform analyses making tissue real parameters evaluation easier. Besides, it can be applied to a quantitative analysis of turbid mediums.

\section{References}

[1] D. Huang, E.D. Swanson, C.P. Lin, J.S. Schuman, W.G. Stinson, W. Chang, M.R. Hee, T. Flotte, C.A. Puliafito, J.G. Fujimoto, Science 254, 1178 (1991).

[2] A.F. Ferher, J. Biomed. Opt. 1, 157 (1996).

[3] M. Wojtkowski, A. Kowalczyk, P. Targowski, I. Gorczyńska, Opt. Appl. 32, 569 (2003).

[4] M. Wojtkowski, V. Srinivasan, T. Ko, J. Fujimoto, A. Kowalczyk, J. Duker, Opt. Expr. 12 , 2404 (2004). http://www.opticsinfobase.org/ abstract . cfm?URI=oe-12-11-2404

[5] I. Gorczyńska, A. Szkulmowska, M. Szkulmowski, P. Targowski, J.J. Kałuzny, M. Wojtkowski, J.G. Fujimoto, A. Kowalczyk, Prog. Biomed. Opt. Imag. Proc. SPIE 5861, 1 (2005).

[6] P. Maciejewicz, J. Kasprzak, D. Kęcik, M. Brodecka, A. Zajacc, in: Proc. Conf. 7 STL 2002, Ed. W. Woliński , Wydawnictwo Uczelniane Politechniki Szczecińskiej 2002, p. 239 (in Polish).

[7] Signal Processing Toolbox User's Guide, MathWorks, Inc., 1988-1998. 